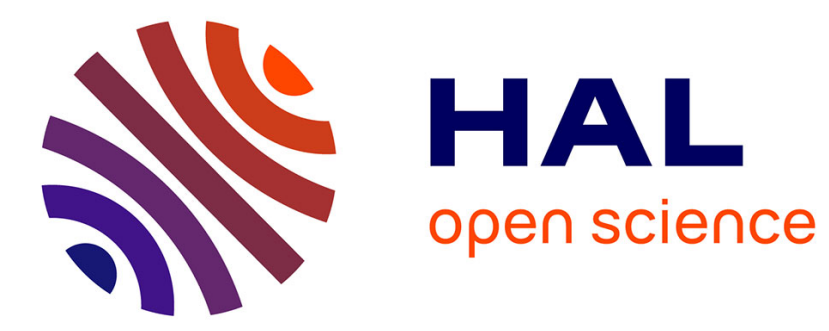

\title{
A bayesian learning of probabilistic relations between perceptual attributes and technical characteristics of car dashboards to construct a perceptual evaluation model
}

Walid Ben Ahmed, Bernard Yannou

\section{- To cite this version:}

Walid Ben Ahmed, Bernard Yannou. A bayesian learning of probabilistic relations between perceptual attributes and technical characteristics of car dashboards to construct a perceptual evaluation model. International Journal of Product Development, 2009, 7 (1-2), pp.47-72. 10.1504/IJPD.2009.022276 . hal-00748729

\author{
HAL Id: hal-00748729 \\ https://hal.science/hal-00748729
}

Submitted on 17 Mar 2013

HAL is a multi-disciplinary open access archive for the deposit and dissemination of scientific research documents, whether they are published or not. The documents may come from teaching and research institutions in France or abroad, or from public or private research centers.
L'archive ouverte pluridisciplinaire HAL, est destinée au dépôt et à la diffusion de documents scientifiques de niveau recherche, publiés ou non, émanant des établissements d'enseignement et de recherche français ou étrangers, des laboratoires publics ou privés. 


\title{
A bayesian learning of probabilistic relations between perceptual attributes and technical characteristics of car dashboards to construct a perceptual evaluation model
}

\author{
Walid Ben Ahmed, Bernard Yannou* \\ Power Train Division, Renault \\ Sce 66101/UET Fiabilité Prévisionnelle \\ DCT/Dépt Métier Fiabilité et MAP, FR CTR A02 370 \\ 67 rue des Bons Raisins, 92500 Rueil-Malmaison, France \\ E-mail: walid.ben-ahmed@ renault.com \\ Laboratoire Genie Industriel, Ecole Centrale Paris \\ Grande Voie des Vignes, 92295 Chatenay-Malabry, France \\ E-mail: bernard.yannou@ecp.fr \\ *Corresponding author
}

\begin{abstract}
Starting from a primary perceptual evaluation of a set of car dashboards, we propose to build a Bayesian network (BN) between perceptual attributes and design attributes. Two types of learning processes may be considered: supervised $\mathrm{BN}$ when the prediction on a targeted attribute must be optimized and unsupervised BN otherwise. These two types of BNs are considered along three design simulation scenarios: the direct scenario which consists of the prediction of a design change impact on customer perceptions, the inverse scenario for fixing design characteristics so as to result in an expected customer perception, and a more realistic combined scenario.
\end{abstract}

Keywords: decision-making; kansei engineering; design synthesis; perceptual evaluation; emotional design; bayesian networks.

Reference to this paper should be made as follows: Ben Ahmed, W.; Yannou, B. $(\mathrm{xxxx})$ 'A bayesian learning of probabilistic relations between perceptual attributes and technical characteristics of car dashboards to construct a perceptual evaluation model', Int. J. Product Development, Vol. X, No. Y, pp.000-000.

Biographical notes: Dr Walid Ben Ahmed received a Ph. D. (2005) in Knowledge Engineering and Management in design from the Industrial Engineering Department at Ecole Centrale Paris (ECP) in 2004. He received a M. Sc. (2001) in design and product system development from ECP (Ecole Centrale Paris). He obtained his degree in mechanical engineering in 2000 at the National Engineering School of Tunis (ENIT). His research interests include complex system modelling, product modelling, reliability management, knowledge engineering and system development in design. He is now in charge of innovation risk management in the Powertrain Engineering Division in Renault company and he is an expert in product reliability.

Bernard Yannou is a full Professor of Industrial and Mechanical Engineering at the Laboratoire Génie Industriel of Ecole Centrale of Paris, France. He received a M. Sc. (1988) in Mechanical Engineering from Ecole Normale Supérieure of 


\section{W. Ben Ahmed, B. Yannou}

Cachan, and M. Sc. (1989) in Computer Science from Paris-6 University. He received a Ph. D. (1994) in Industrial Engineering from Ecole Normale Supérieure of Cachan. His research interests are centred on the preliminary stages of product design: defining the design requirements, synthesizing product concepts, rapid evaluation of product performances, preference aggregation of the product and the project performances for the supervision of the design process, subjective and perceptual evaluation.

\section{Introduction}

A design process can be seen as an iterative and complex process guided by a final and ultimate objective, which is to make the developed product fitting the customer aspirations. Hence, predicting customers' satisfaction level when one develops a new product is fundamental. Designers need tools to help them understanding customers' needs and thereby predicting their appreciation level of a new product. During a design process, designers are facing two main issues that we explain through the two following questions. The first one is a question of simulation of performances (deductive reasoning), denoted hereafter direct/analysis: what is the impact of a given decision related to the design parameters (i.e. technical and/or functional parameters) on the final customer perception. The second is more narrowly related to the design goal itself, starting from the need (expected performances or perceived impacts) and inducing a satisfactory design solution, denoted hereafter the inverse/synthesis scenario: what are the optimal levels of design technical attributes that can meet a given expected perception by a customer?

Certainly, the two questions are connected and can be handled if the correlation between the design attribute levels and the customer perceived impact levels is known. This correlation can be learnt through experience. In other words, experienced engineers are capable to convert a subjective expected perception expressed by customers in concrete technical design attribute levels. However, their suggestions remain subjective and generally difficult to explain. The second way to rationalize this correlation is to construct an analytic predictive model. In this paper, we propose to construct such a model using data mining learning techniques and more specifically Bayesian Networks (BNs).

As a case study, we propose to use customers' evaluations of perceptual attribute levels of existing car dashboards in order to predict the customer perception of new car dashboards by assuming their technical attributes or main design parameters (design analysis situation). Conversely, we also propose to advise designers on values of dashboard physical attributes so as to better ensure meeting given customer perceptions on these dashboards (design synthesis). Henceforth, we call technical (or physical) attribute any parameter that deals with the design characteristics (shape type, number of colors, subsystem location, average curvature radius of shapes, etc.). In other words, technical attributes are parameters that a designer can tune to change and ultimately optimize his design. We call perceptual (or subjective) attributes all variables that deal with the customer evaluation of the design (comfort, novelty, cultural values). In the following, perceptual attributes are assessed by a set of customer in an non-hedonistic 
A bayesian learning between perceptions and technical characteristics

way, i.e. perceptual attributes are assessed on an absolute scale independently of personal preferences (as much as possible). This scale is automatically built using pairwise comparison techniques which are widely presented in (Petiot and Yannou, 2004, Yannou and Petiot, 2004, Yannou, 2007) and which are not the subject of this paper.

Various scientific approaches relating physical attributes and perceptual attributes have been gathered by Japanese researchers under the name of Kansei Engineering, ours may be considered as such. This research aims at exploring the structure of emotions by building a database on consumer feelings. From the consumer's point of view, a forward mapping process from perceptual words to design elements is established, and from the designer's point of view, a backward process from drawings to perceptual words is proposed (Nagamachi, 1995, Nagamachi, 2002). These two processes exactly correspond to, respectively, the aforementioned synthesis and analysis use scenarios. Some methods of category classification methods based on the Semantic Differential Method (SDM) have been used for the design of car interiors for example (Jindo and Hirasago, 1997). More sophisticated methods based on genetic algorithms, neural networks or fuzzy logic have been applied to ensure mappings between perceptual words and design elements, but these systems are often opaque for designers and consumers. A semantic transformation method for automotive form design is proposed in (Hsiao and Wang, 1998), allowing an automatic regulation of the shape with respect to the required image required. The aim of this paper is to study the use of a still rarely used technique in design, namely the Bayesian Networks. Its main advantages compared to other techniques are that they are not opaque to the designer and that they can be easily used in compound analysis/synthesis scenarios, complying with actual design problems.

After presenting Bayesian Networks in section 2, the collect protocol for perceptual data is briefly evoked in section 3. Section 4 describes the model building and the use scenarios. Section 5 is a discussion of the present approach.

\section{Theoretical background: Bayesian network}

Consider " $A "=$ "use comfort" $=\{$ weak, good $\}$ as one of the perceptual attributes and " $B "="$ control button shape" $=\{$ circular, square $)$ as one of the physical attributes characterizing the dashboards. The double functionality of our model (direct and inverse) is possible thanks to Bayesian Networks (BNs), which are based on the Bayes theorem given by formula (1):

$$
\mathrm{P}(\mathrm{A} / \mathrm{B})=\frac{\mathrm{P}(\mathrm{B} / \mathrm{A}) \cdot \mathrm{P}(\mathrm{A})}{\mathrm{P}(\mathrm{B})}
$$

BNs are directed acyclic graphs used to represent uncertain knowledge in Artificial Intelligence (Jensen, 1996). A BN is defined as a couple: $B N=(S, P)$, where:

- $S=(N, A)$ represents the structure (i.e. the graph);

- " $N$ " is a set of nodes. Each node represents a discrete variable $X$ having a finite number of mutually exclusive states (modalities). In our case study, $X$ may be a perceptual attribute as well as a technical attribute; 
- " $A$ " is a set of edges; the relation " $N_{1}$ is a parent of $N_{2}$ " is represented by an edge linking $N_{l}$ to $N_{2}$. In our case study, an edge may be interpreted as a causal relation.

- $\quad P$ represents a set of probability distributions that are associated to each node. When a node is a root node (i.e. it does not have a parent), $P$ corresponds to the probability distribution over the node states. When a node is not a root node, i.e. when it has some parent nodes, $P$ corresponds to a conditional probability distribution that quantifies the probabilistic dependency between that node and its parents. It is represented by a Conditional Probability Table (CPT).

Because a Bayesian network is a complete model for the attributes and their relationships, it can be used to answer probabilistic queries about them. For example, the network can be used to find out updated knowledge of the state of a subset of attributes when other attributes (the evidence attributes) are observed. This process of computing the posterior distribution of attributes given evidence is called probabilistic inference. Various inference algorithms can be used to compute marginal probabilities for each unobserved node given information on the states of a set of observed nodes. The most classical one relies on the use of a junction tree (see (Jensen, 1996), pp. 76). Inference in BN (Huang and Dawiche, 1996) allows then taking any state attribute observation (an event) into account to update the probabilities of the other attributes. Without any event observation, the computation is based on a priori probabilities. When observations are given, this knowledge is integrated into the network and all the probabilities are updated accordingly.

The use of Bayesian networks in industry is continually growing up especially in risk management fields, in marketing, and generally in domains where there is uncertainty and thereby a need to predict a complex behavior such as in a decision making process.

Many commercial tools provide users with HMI allowing graphical representation of Bayesian networks to model expert knowledge. They are also providing users with a panel of supervised and unsupervised learning algorithms to extract automatically knowledge from data bases (see section 4).

\section{Data preparation}

The data colleting protocol has been described in (Yannou, 2007) and already experimented on another case study in (Petiot and Yannou, 2004, Yannou and Petiot, 2004). Ten dashboards, displayed in Figure 1 (AUDI A2, CITROEN C2, FIAT Idea, LANCIA Ypsilon, NISSAN Micra, PEUGEOT 206, RENAULT Clio, RENAULT Modus, TOYOTA Yaris, VW Polo), are evaluated by 11 customers. Each dashboard is represented by a 19-dimensional feature vector, $X=\left\{x_{1}, \ldots, x_{19}\right\}$, depicting 19 measurements made on the dashboard from 19 attributes that we present hereafter in more details: 8 technical (or physical) attributes and 11 perceptual attributes. 
A bayesian learning between perceptions and technical characteristics
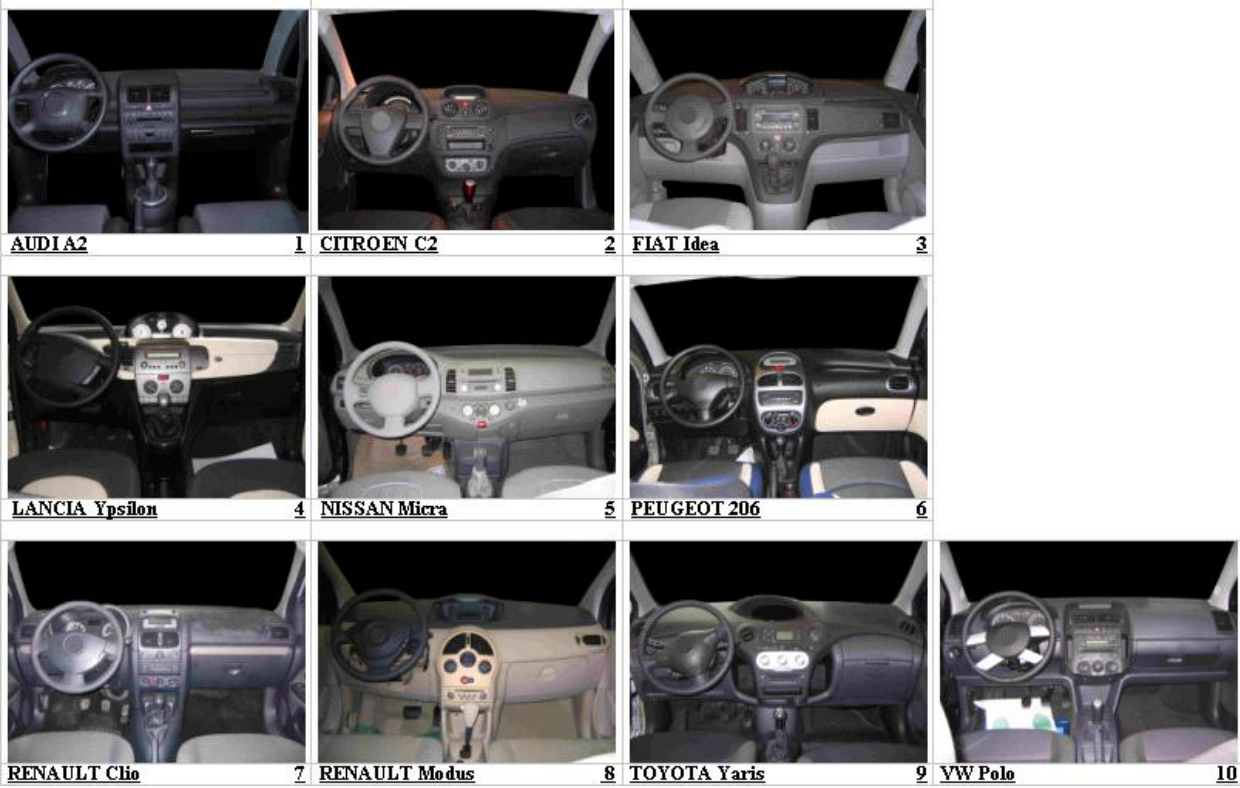

Figure 1 The 10 dashboards evaluated by customers

We defined a set of 8 technical (or physical) attributes characterizing the dashboards with corresponding modalities (two at least, but the number may increase): the "Speedometer dial position" $=\{$ behind steering wheel, at the center of the dashboard $\}$, "Display layout" $=\{$ Analogue, Digital $\}$, "Air conditioner control" $=\{$ Button, Other $\}$, "Air vent shape $"=\{$ Rounded, Square $\}, "$ Dashboard color $"=\{$ Single color, Two colors $\}, "$ Aerator shape"=\{Rounded, Square $\}$, "Arrangement space" $=\{$ Many, Few $\}$ and "Style layout $"=\{$ Curved lines, Straight lines $\}$. The characterization of the 10 dashboards according to the technical attributes is objective and do not depend on the preference of customers. It is presented in table 1.

We also defined a set of 11 perceptual attributes, which describe the customer perception of the "Space organization", "Control button comprehensibility", "Aerator lay-out", "Arrangement space", "Comfort", "Simplicity", "Sportive lay-out", "Masculinity lay-out", "Quality", "Novelty" and "Harmony" (see (Harvey, 2005) for details on attributes) The customer evaluations of the dashboard perceptual attribute levels is made in qualitatively pairwise comparing the 10 dashboards under each of the 11 perceptual attributes (see (Limayem and Yannou, 2004) for mathematical details). It leads to 11 normalized score vectors. The advantage of this method is that the value scale is automatically built thanks to the pairwise comparison mechanism without the need to define a specific metrics (for instance, a score of 0.1 for the "Masculinity lay-out" means much more feminine than a score of 0.3 ). Next, each normalized score vector (the scores sum is 1) is transformed to fit into a standard scale of [0, 20]. Finally, continuous attribute levels are projected into discrete categories: $[0,5]=$ Very low, $[6,10]=$ Low, $[10$, 14]=Medium, [15, 17]=High, [18, 20]=Very high. As 11 customers have participated to this study, a $110 \times 19$ matrix is then constructed: rows $=10$ dashboards $\times 11$ customers, columns $=8$ technical $\& 11$ perceptual attributes. 
W. Ben Ahmed, B. Yannou

Table 1 The physical characterization of the 10 dashboards

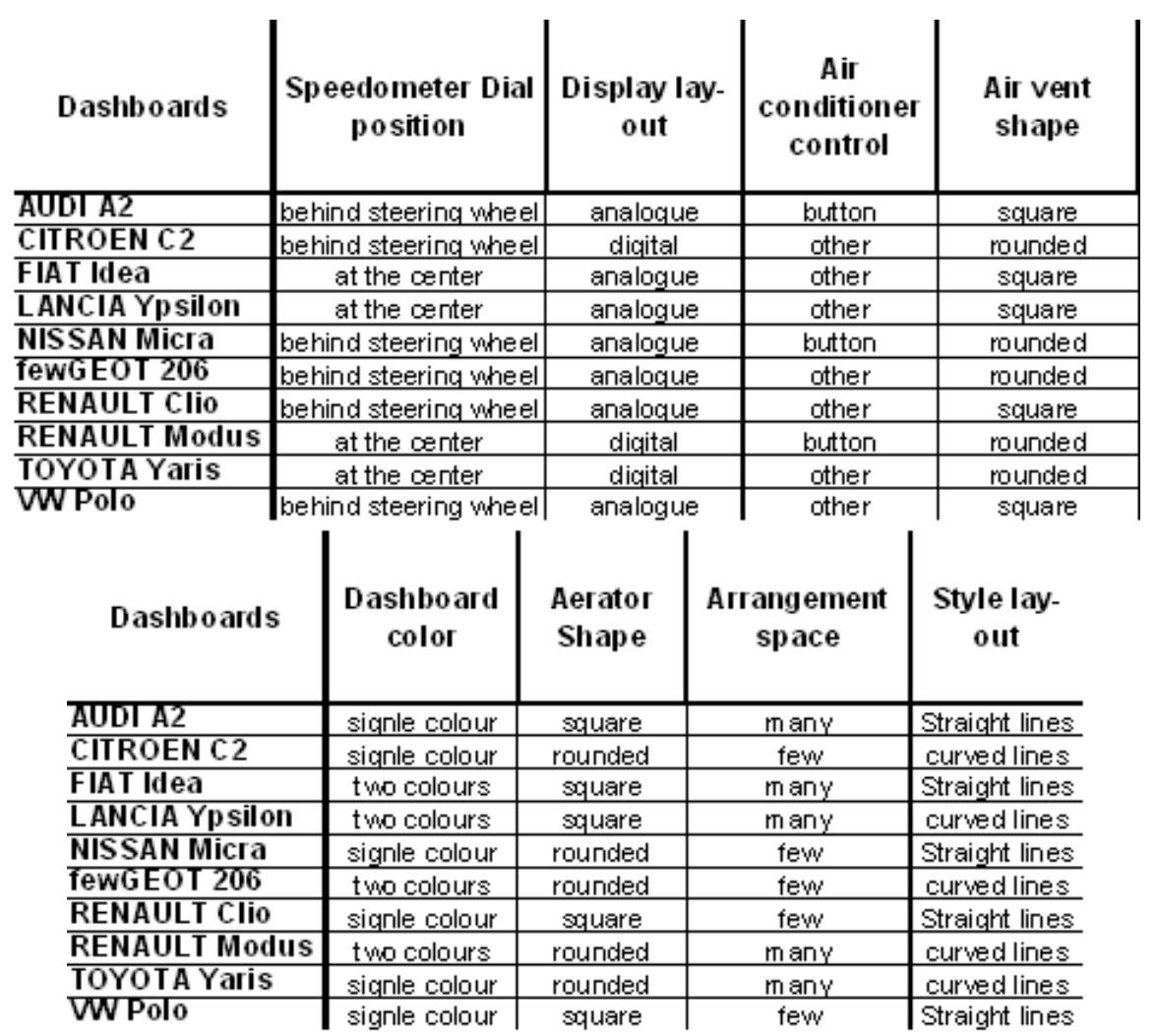

We are aware that data discretization has some drawbacks: for instance, although 13 is as similar to 14 as 15 , they belong to different categories. In spite of this limitation, results we obtained are satisfactory (cf. section 4.3). In an underway work, we apply bayesian network directly on data stemmed from pairwise comparisons and thereby we do not need to descritize data. The data discretization issue may also be handled by the use of fuzzy logic. Applying bayesian learning on fuzzy data would then be an interesting research perspective.

\section{Kansei model construction using bayesian network learning}

The Kansei model elaboration consists in constructing a $\mathrm{BN}$ in which a node represents an attribute (technical or subjective) and edges represent direct probabilistic relations between the connected attributes. Attributes are defined over several states (or modalities) as we explained previously. The statement of the probabilistic relations between attributes may be performed by experts and/or automatically extracted from the data set we presented above by the use of supervised and/or unsupervised learning techniques. In this paper we use automatic learning of Kansei bayesien model from data.

There are several techniques for learning Bayesian networks from data (see (Spirtes et al., 2000, Neapolitan, 2003, Buntine, 1996, Jordan, 1998, Naïm et al., 1999) for an overview). Two main techniques may be distinguished. The first one is a score-based 


\section{A bayesian learning between perceptions and technical characteristics}

method and the other is a constraint-based method. The constraint-based method employs statistical tests on the data set for deciding the existence of edges in the Bayesian network. The accuracy of the constraint-based method strongly depends on the size of the data set. A huge data set can provide a more accurate statistical independence test. In this paper, we use a small data set, so we adopt the score-based approach.

Methods to learn Bayesian networks from data often consist of two components. The first component is a score (i.e. a cost function) which is used to evaluate how well the learned model fits the data. The second component is a learning algorithm (i.e. a structure search strategy) which is used to identify one or more network structures with high scores by searching through the space of possible network structures.

- The score function: there have been some scores proposed for learning Bayesian networks. These includes the AIC score (Akaike, 1974), the BIC score (Schwarz, 1978), the K2 score (Cooper and Herskovits, 1992), the BDe score (Heckerman et al., 1995), the GU score (Mehmet and Gregory, 2002) and the MDL (Minimum description length) score (Lam and Bacchus, 1994, Rissanen, 1986, Bouckaert, 1993, Suzuki, 1993). Remco R. Bouckaert (Bouckaert, 1993) indicates that the performance of learning Bayesian network structure using MDL score is slightly better than the performance of the other scores. In this paper, we use the MDL, which is an information-theoretic criterion that favors models which provide the shortest description of the training data. This description includes both the description of the model and the description of the data given the model. Formally, given a Bayesian network $\mathrm{BN}=(\mathrm{S} ; \mathrm{P})$, and a training data set $\mathrm{D}$, the MDL score of $\mathrm{BN}$ is defined as $\operatorname{Score}_{M D L}(B N ; D)=\operatorname{MDL}(B N)+M D L(D \backslash B N)$. Without going into details of MDL derivation, we just note here that the first term of the MDL score is the description length of a Bayesian network, i.e. the number of bits required to encode the network parameters, while the second term is the negative log likelihood of the model $\mathrm{BN}$ given data $\mathrm{D}$, which gives the number of bits needed to describe $\mathrm{D}$ when using BN.

- Structure searching strategy: the number of possible network structures (NS) grows exponentially with the number of nodes $(n)$ (Robinson, 1977, Friedman and Koller, 2003) after formula (2):

$$
N S(n)=\sum_{i=1}^{n}(-1)^{i+1}\left(\begin{array}{l}
n \\
i
\end{array}\right) 2^{i(n-1)} N S(n-i) \text { for } n>1
$$

For example $N S(5)=29281$ and $N S(10)=4.2 \times 10^{18}$ (Robinson, 1977). Therefore, most algorithms for learning Bayesian networks are heuristic search algorithms. Some examples are the K2 algorithm (Cooper and Herskovits, 1992), the Structure EM algorithm (Friedman, 1998), the Greedy Equivalent Search (GES) algorithm (Meek, 1997, Chickering, 2002a, Chickering, 2002b), and so on.

We propose in this paper two types of Kansei engineering models: an unsupervised model and a supervised model. 


\section{W. Ben Ahmed, B. Yannou}

\subsection{Unsupervised Kansei model construction}

An unsupervised Kansei model consists in an unsupervised learning of the whole set of probabilistic relationships existing within the data and especially between perceptual attributes and technical attributes. We used the SopLEQ technique, which is a quick search based on the whole dataset. It uses a cost function based on the MDL score and a structure search method based on equivalent model classes (Jouffe and Munteanu, 2001, Munteanu and Bendou, 2001, Jouffe, 2002). The description of SopLEQ as well as its advantages compared to the other search techniques is pointed out in (Jouffe and Munteanu, 2001, Munteanu and Bendou, 2001, Jouffe, 2002). Figure 2 represents the constructed BN without any user modifications.

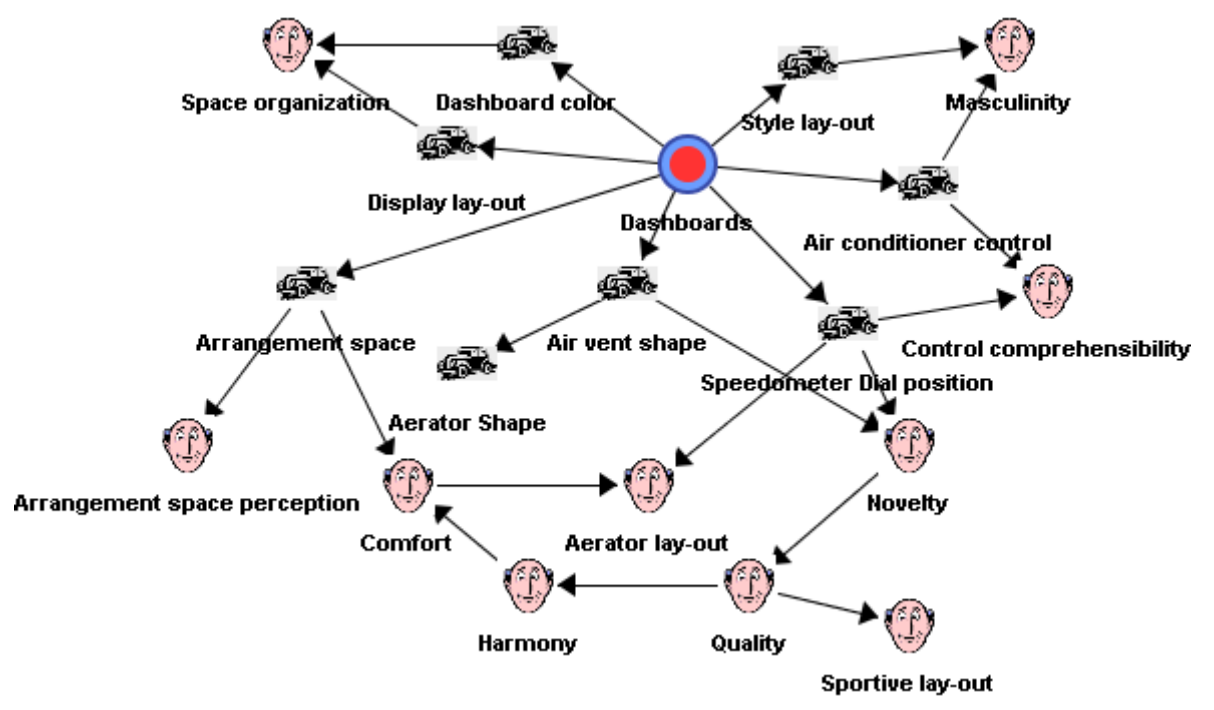

Figure 2 Unsupervised learning to identify probabilistic relationships within the data (i.e. between dashboard physical - car icon - and perceptual - face icon - attributes)

Edges in this bayesian network can be interpreted as causal relationships. For instance, according to Figure 2, the subjective attribute "Novelty" depends on the two physical attributes "Air vent Shape" and "Speedometer position". Each relation (i.e. edge) is expressed through a conditional probability table, which is automatically computed. For example, the relation between "Novelty", "Air vent Shape" and "Speedometer position" is represented through Table 2 . 
A bayesian learning between perceptions and technical characteristics

Table 2 Conditional probabilities representing the causal relation between "Air vent Shape", "Speedometer position" and "Novelty". According to this table : $\mathrm{P}($ novelty $=$ very low $\mid$ Speedometer dial position=at the center $\&$ Air vent shape=rounded $)=13,6 \%$

\begin{tabular}{|c|c|c|c|c|c|c|}
\hline \multirow{2}{*}{$\begin{array}{l}\text { Speedometer dial } \\
\text { position }\end{array}$} & \multirow{2}{*}{$\begin{array}{l}\text { Aerator } \\
\text { shape }\end{array}$} & \multicolumn{5}{|l|}{ Novelty } \\
\hline & & Very low & Low & Medium & High & Very High \\
\hline \multirow[t]{2}{*}{ At the centre } & Rounded & 13.6 & 36.4 & 31.8 & 9.1 & 9.1 \\
\hline & Square & 27.3 & 36.4 & 27.3 & 0.0 & 9.1 \\
\hline \multirow{2}{*}{$\begin{array}{l}\text { Behind } \\
\text { wheel }\end{array}$} & Rounded & 24.2 & 60.6 & 9.1 & 6.1 & 0.0 \\
\hline & Square & 75.8 & 24.2 & 0.0 & 0.0 & 0.0 \\
\hline
\end{tabular}

We notice here that the constructed model (cf. Figure 2) allows identifying three types of relationships:

- Relationships within technical attributes. For example, "Air vent shape" has a direct impact on the "Aerator shape".

- Relationships within perceptual attributes. For example, "harmony" perception has a direct impact on "comfort" perception.

- Relationships between technical and perceptual attributes. For example, the two physical attributes "Air vent Shape" and "Speedometer position" have an impact on the "Novelty" perception.

\subsection{Supervised Kansei model construction}

Instead of learning a Bayesian network representing all the probabilistic relations that hold in the data set such as we performed in the previous paragraph, it is possible to apply supervised learning algorithms to characterize a specific target attribute. This technique allows identifying the attributes that really characterize the target attribute. The target attribute may be a perceptual attribute such as "Novelty" when user looks for understanding and characterizing a customer satisfaction. It may also be a technical attribute when user has to perform a decision about that attribute in order to optimize it.

When the user looks for characterizing a target attribute (e.g. novelty), the supervised learning is more relevant to apply than the unsupervised learning. Indeed, the supervised learning focuses the learning on identifying relations between the target attribute and the other attributes instead of learning all the probabilistic relations that hold in the data set. Thereby, the result of a supervised learning is more accurate with respect to the target attribute than the result given by an unsupervised learning. For instance, according to the model presented in Figure 3, the main physical attributes really influencing a customer perception of a dashboard "novelty" are the "speedometer dial position", the "dashboard color" and the "style lay-out". This is quite different compared to the result of the unsupervised model (cf. Figure 2), which suggests that the "speedometer position" and the "air vent shape" are the technical attributes that directly influence the "novelty" perception. In section 4.3 , we show through a numerical evaluation that the supervised learning provides more accurate results than the unsupervised learning when one looks for characterizing a given target attribute. 
Unsupervised and supervised models are complementary: through a global optimization, the unsupervised technique allows having an idea about all relationships that hold in the data set. On the other hand, the supervised technique allows refining the characterization of a target attribute.

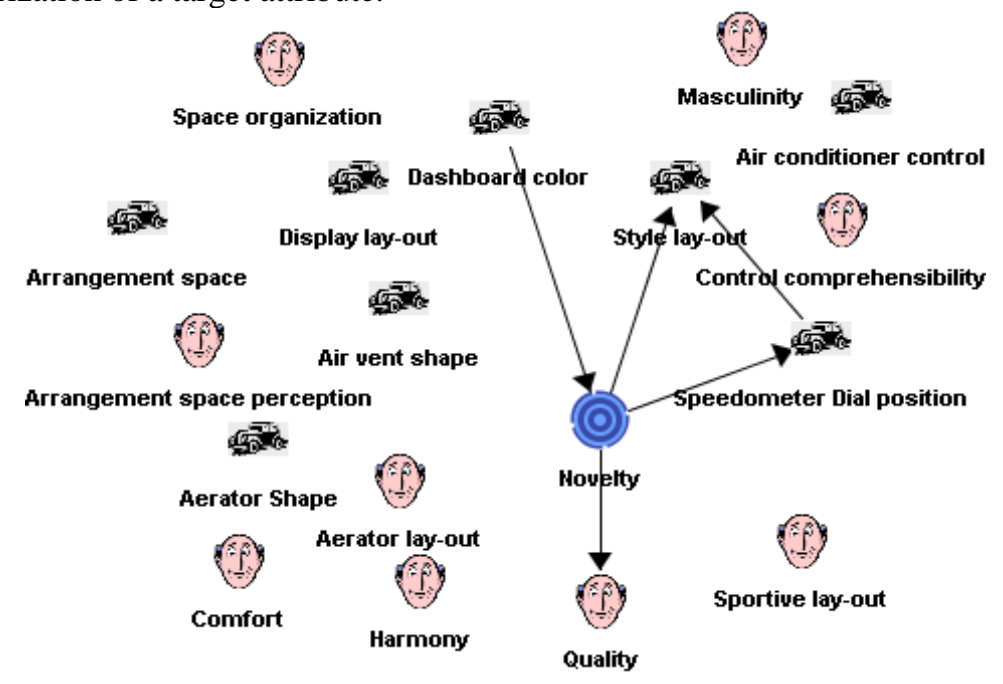

Figure 3 Supervised learning to characterize a target attribute (e.g. "Novelty"). This model shows that the main physical attributes really influencing the "novelty" perception of a dashboard are the "speedometer dial position", the "dashboard color" and the "style lay-out". The model also points out a probabilistic relation between the perceptual attributes "novelty" and "quality perception".

We tested several algorithms to construct the supervised model presented in Figure 3 (Markov Blanket and Augmented Markov Blanket). All these algorithms give the same result. The presentation as well as the comparison of these algorithms is out of the purpose of this paper (see (BayesiaLab, 2006) for more details).

In section 4.4, we show how unsupervised and supervised learnt models, such those we constructed respectively in section 4.1 and 4.2 , are used by a designer in order to evaluate design solutions or/and identifying the best technical solutions so as to optimize a target attribute of a design.

\subsection{Model evaluation}

We distinguish two types of model evaluation tasks: expert evaluation and numerical evaluation.

The expert evaluation consists in verifying that the model constructed has identified the evident relationships commonly known by the domain experts. Otherwise, an expert may use another score function, another structure search strategy and/or reconsider the data-preprocessing step (i.e. performing another discretization of the data).

In this paper, we propose two assessing methods according to the type of the Kansei bayesian mode (i.e. unsupervised or supervised). 
A bayesian learning between perceptions and technical characteristics

\subsubsection{Numerical assessing of unsupervised Kansei model}

The numerical accuracy of an unsupervised model is written as: $P(D \mid B N)$ meaning the probability of the data given the Bayesian Network $(\mathrm{BN})$. It is more common to use the log likelihood of BN given D provided by formula (3):

$$
\log _{2}\left(P(D \mid B N)=\sum_{i=1}^{n} \sum_{j=1}^{Q i} \sum_{k=1}^{R i}-\left(N_{i j k} / N\right) * \log \left(N_{i j k} / N_{i j}\right)\right.
$$

where:

- $U$ is the set of attributes $\left\{X_{1}, X_{2}, \ldots . X_{n}\right\}, n>=1$,

- $\quad N$ is the total number of attributes,

- $X_{i}$ is an attribute which takes values from $\left\{X_{i 1}, X_{i 2} \ldots\right\}$,

- $R_{i}$ is the total number of values of $X_{i}$,

- $\quad D$ is the data set over $U$,

- $B N$ is a Bayesian network structure over $U$,

- $\quad N$ is a number of instances in $D$,

- $Q_{i}$ is the total number of value combinations of $P_{i}$ in $B N$,

- $\quad N_{i j k}$ is the number of cases in $D$ in which $X_{i}=X_{i k}$ and $P_{i}=W_{i j}$.

The log likelihood measures how many bits are needed to describe D based on the probability distribution P. It also has a statistical interpretation: the higher the $\log$ likelihood, the closer BN is to model the probability distribution in the data D.

To assess our unsupervised Kansei model, we divided the data into two subgroups, a training set $\left(D_{\text {training }}=80 \%\right.$ of the data set $)$ and a testing set $\left(D_{\text {test }}=20 \%\right.$ of the data set $)$. We selected the testing set so as to be the most representative as possible with respect to the original data. Next, we learned a BN using the training set. Then, we compared the $\log$ likelihood of $\mathrm{BN}$ given $D_{\text {training }}$ and the $\log$ likelihood of $\mathrm{BN}$ given $\mathrm{D}_{\text {test }}$. The model is acceptable if the two values are close, i.e. $\log \left(P\left(D_{\text {training }} / B N\right)\right) \infty \log \left(P\left(D_{\text {test }} / B N\right)\right)$. This means that the model is able to represent unseen data (i.e. $\left.\mathrm{D}_{\text {test }}\right)$.

$\log \left(P\left(D_{\text {training }} / B N\right)\right)=21.75$ and $\log \left(P\left(D_{\text {test }} / B N\right)\right)=24.38$. The model fits at least as well as good to testing set than to training set.

\subsubsection{Numerical assessing of a supervised Kansei model}

As we stressed in section 4.2, when the user looks for characterizing a target attribute (e.g. novelty), supervised learning is more relevant to apply than unsupervised learning. In this section, we show through a numerical evaluation that the supervised learning gives also more accurate results than unsupervised learning when one looks for characterizing a target attribute.

To do so, we consider a supervised model and an unsupervised models as classifiers: the $m$ modalities of the target attribute are considered as $m$ classes $\left\{C_{l}, \ldots, C_{m}\right\}$ and the role of the bayesian network is to assign an unknown dashboard $X$ to a class $C_{i}$ according to the measurements made on the dashboard. 
We consider the two models (supervised and unsupervised) we presented in sections 4.1 and 4.2. They have been constructed from the same training set $D_{\text {training. Then we }}$ compare their ability to predict the novelty class (i.e. very low, low, medium, high or very high) of a set of dashboards $D_{\text {test }}$ that did not participate in the models learning. We define the sensitivity and precision notions to compare the models accuracy by formula (4):

$$
\text { sensitivity }=\frac{t_{-} \text {pos }}{\text { pos }}, \quad \text { precision }=\frac{t_{-} \text {pos }}{t_{-} p o s+f_{-} p o s}
$$

where:

- $\quad$ pos is the number of positives: samples that really belong to class $C_{i}$ (the sum of rows in the occurrency table of Table 3,

- $\quad t_{\text {p pos }}$ is the number of true positives: samples that belong to $C_{i}$ and were correctly classified (the diagonal of the occurrency table of Table 3 ),

- $\quad f_{\_}$pos is the number of false positives: samples that do not belong to $C_{i}$ and were uncorrectly classified in $C_{i}$ (numbers that do not belong to the diagonal of the occurrency table of Table 3).

Table 3 regroups the concurrency tables, the sensitivity tables and the precision tables that allow us to compare supervised and unsupervised models accuracy. According to these tables, supervised model is more accurate to predict the "novelty" of a dashboard characterized by the 18 other attributes. 
A bayesian learning between perceptions and technical characteristics

Table 3 The comparison of the sensitivity and the precision of supervised and unsupervised Kansei models. According to this table, supervised model is more accurate to predict the "novelty" of a dashboard characterized by the 18 other attributes.

\begin{tabular}{|c|c|c|c|c|c|c|}
\hline \multicolumn{7}{|c|}{ Prediction obtained using the supervised model } \\
\hline \multicolumn{7}{|c|}{ Occurrency table } \\
\hline \multirow{2}{*}{\multicolumn{2}{|c|}{ Novelty }} & \multicolumn{5}{|c|}{ Pos } \\
\hline & & Very low (2) & Low (9) & Medium (4) & High (2) & \begin{tabular}{|l|} 
Very high (1) \\
\end{tabular} \\
\hline \multirow{5}{*}{ 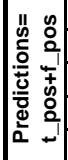 } & Very low (4) & 2 & 2 & 0 & 0 & 0 \\
\hline & Low (9) & 0 & 6 & 2 & 1 & 0 \\
\hline & Medium (4) & 0 & 1 & 2 & 1 & 0 \\
\hline & High (0) & 0 & 0 & 0 & 0 & 0 \\
\hline & Very high (1) & 0 & 0 & 0 & 0 & 1 \\
\hline \multicolumn{7}{|c|}{ Sensitivity table $=t \_p o s / p o s=x i j / s u m \_c o l u m n$} \\
\hline \multirow{2}{*}{\multicolumn{2}{|c|}{ Novelty }} & \multicolumn{5}{|c|}{ Pos } \\
\hline & & Very low (2) & Low (9) & Medium (4) & High (2) & Very high (1) \\
\hline \multirow{5}{*}{ 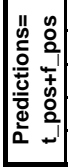 } & Very low (4) & $50.0 \%$ & $50.0 \%$ & $0.0 \%$ & $0.0 \%$ & $0.0 \%$ \\
\hline & Low (9) & $0.0 \%$ & $66.66 \%$ & $22.22 \%$ & $11.11 \%$ & $0.0 \%$ \\
\hline & Medium (4) & $0.0 \%$ & $25.0 \%$ & $50.0 \%$ & $25.0 \%$ & $0.0 \%$ \\
\hline & High (0) & $0.0 \%$ & $0.0 \%$ & $0.0 \%$ & $0.0 \%$ & $0.0 \%$ \\
\hline & Very high (1) & $0.0 \%$ & $0.0 \%$ & $0.0 \%$ & $0.0 \%$ & $100.0 \%$ \\
\hline \multicolumn{7}{|c|}{ Precision table $=x i j /$ sum_lign $=t \_p o s / t \_p o s+f \_p o s$} \\
\hline \multirow{2}{*}{\multicolumn{2}{|c|}{ Novelty }} & \multicolumn{5}{|c|}{ Pos } \\
\hline & & Very low (2) & Low (9) & Medium (4) & High (2) & Very high (1) \\
\hline \multirow{5}{*}{ 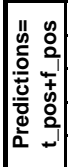 } & Very low (4) & $100.0 \%$ & $22.22 \%$ & $0.0 \%$ & $0.0 \%$ & $0.0 \%$ \\
\hline & Low (9) & $0.0 \%$ & $66.66 \%$ & $50.0 \%$ & $50.0 \%$ & $0.0 \%$ \\
\hline & Medium (4) & $0.0 \%$ & $11.11 \%$ & $50.0 \%$ & $50.0 \%$ & $0.0 \%$ \\
\hline & High (0) & $0.0 \%$ & $0.0 \%$ & $0.0 \%$ & $0.0 \%$ & $0.0 \%$ \\
\hline & Very high (1) & $0.0 \%$ & $0.0 \%$ & $0.0 \%$ & $0.0 \%$ & $100.0 \%$ \\
\hline \multicolumn{7}{|c|}{ Prediction obtained using the unsupervised model } \\
\hline \multicolumn{7}{|c|}{ Occurrency table } \\
\hline \multirow{2}{*}{\multicolumn{2}{|c|}{ Novelty }} & & & Pos & & \\
\hline & & Very low (2) & Low (9) & Medium (4) & High (2) & Very high (1) \\
\hline \multirow{5}{*}{ 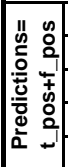 } & Very low (4) & 1 & 3 & 0 & 0 & 0 \\
\hline & Low (9) & 1 & 6 & 4 & 2 & 1 \\
\hline & Medium (4) & 0 & 0 & 0 & 0 & 0 \\
\hline & High (0) & 0 & 0 & 0 & 0 & 0 \\
\hline & Very high (1) & 0 & 0 & 0 & 0 & 0 \\
\hline \multicolumn{7}{|c|}{ Sensitivity table $=t \_p o s / p o s=x i j / s u m \_c o l u m n$} \\
\hline \multirow{2}{*}{\multicolumn{2}{|c|}{ Novelty }} & & & Pos & & \\
\hline & & Very low (2) & Low (9) & Medium (4) & High (2) & Very high (1) \\
\hline \multirow{5}{*}{ 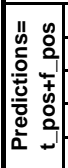 } & Very low (4) & $25.0 \%$ & $75.0 \%$ & $0.0 \%$ & $0.0 \%$ & $0.0 \%$ \\
\hline & Low (9) & $7.14 \%$ & $42.85 \%$ & $28.57 \%$ & $14.28 \%$ & $7.14 \%$ \\
\hline & Medium (4) & $0.0 \%$ & $0.0 \%$ & $0.0 \%$ & $0.0 \%$ & $0.0 \%$ \\
\hline & High (0) & $0.0 \%$ & $0.0 \%$ & $0.0 \%$ & $0.0 \%$ & $0.0 \%$ \\
\hline & Very high (1) & $0.0 \%$ & $0.0 \%$ & $0.0 \%$ & $0.0 \%$ & $0.0 \%$ \\
\hline \multicolumn{7}{|c|}{ Precision table $=x i j / s u m \_l i g n=t \_p o s / t \_p o s+f \_p o s$} \\
\hline & Novelty & & & Pos & & \\
\hline & & Very low (2) & Low (9) & Medium (4) & High (2) & Very high (1) \\
\hline & Very low (4) & $50.0 \%$ & $33.33 \%$ & $0.0 \%$ & $0.0 \%$ & $0.0 \%$ \\
\hline ¿ै ؛ & Low (9) & $50.0 \%$ & $66.66 \%$ & $100.0 \%$ & $100.0 \%$ & $100.0 \%$ \\
\hline 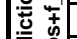 & Medium (4) & $0.0 \%$ & $0.0 \%$ & $0.0 \%$ & $0.0 \%$ & $0.0 \%$ \\
\hline 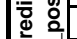 & High (0) & $0.0 \%$ & $0.0 \%$ & $0.0 \%$ & $0.0 \%$ & $0.0 \%$ \\
\hline & Very high (1) & $0.0 \%$ & $0.0 \%$ & $0.0 \%$ & $0.0 \%$ & $0.0 \%$ \\
\hline
\end{tabular}




\subsection{Model simulation}

As we pointed out in the introduction, three main use scenarios of our BN model are possible. We present each of them with explaining examples in the following subsections.

\subsubsection{Direct/analysis scenario}

A design process can be perceived as a decision process during which the designer tunes a set of technical attributes in order to satisfy a required predefined performance. Before carrying out any decision (i.e. technical choice), the designer analyzes the impact of his choice on the other attributes (perceptual as well as physical). The direct/analysis scenario allows answering the question "what is the probable impact of the choice related to physical attributes on the other design attributes and especially on the perceptual attributes". In a sense, this scenario consists in observing a technical attribute and analyzing its impact on the other attributes. It may typically be used to compare different technical solutions.

The two types of models, unsupervised learnt model (Figure 2) and supervised learnt model (Figure 3), may be used to help a designer applying this scenario.

\section{(a) The use of a supervised learnt model in a direct scenario}

In a direct use scenario, the input of a supervising learnt model is a technical (or physical) attribute. Let us take the speedometer dial position as an example. Analyzing the impact of that attribute consists in performing a supervised learning of its relationship with the other attributes.

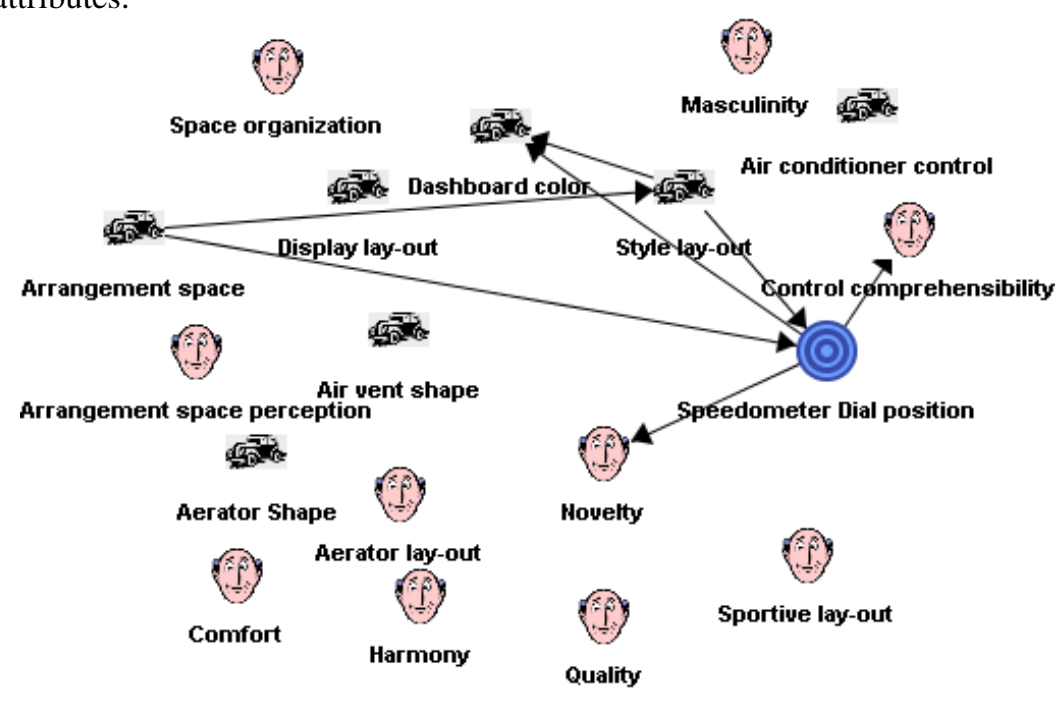

Figure 4 Supervised learning to characterize the relationship of a technical attribute (e.g. "Speedometer dial position") with other technical attributes and its impact on the perceptual attributes. 
A bayesian learning between perceptions and technical characteristics

The model presented in Figure 4 allows identifying two types of relations:

- The interactions of the technical target attribute, i.e. the "speedometer dial position", with the other technical attributes. Figure 4 shows that the "speedometer dial position" has probabilistic correlations with the "dashboard arrangement space", the "dashboard color" and the "dashboard style lay-out". In other words, a change of the speedometer dial position may impact the arrangement space attribute and the choice of the dashboard color and/or the choice of the style lay-out. For instance, the model states that positioning the speedometer at the center of the dashboard may increase the arrangement spaces (cf. Figure 5). It also states that, generally, a dashboard whose speedometer dial is positioned at the center is likely to have curved lines style lay-out and two colors (cf. Figure 5).

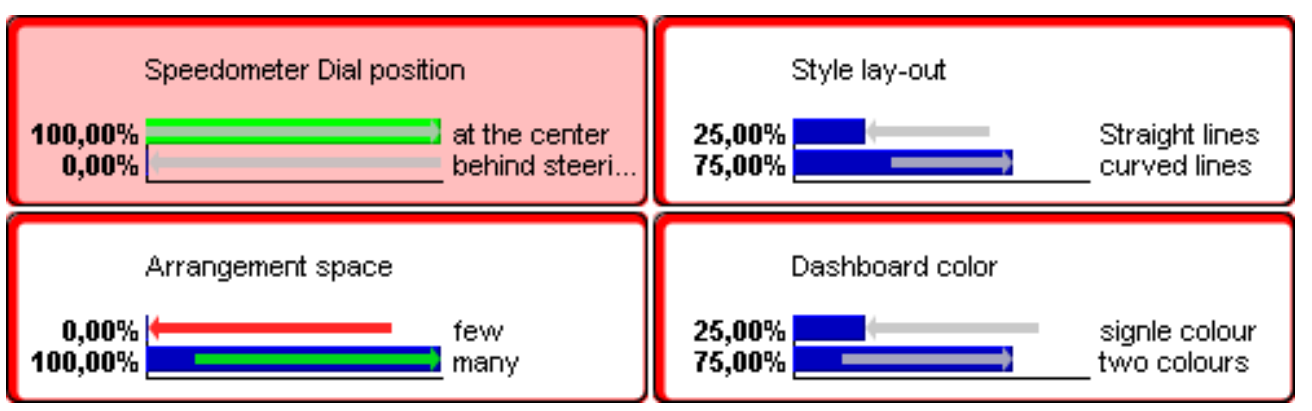

Figure 5 The impact of the choice "“'Speedometer dial position=at the center" on the other technical attributes (The arrows indicate the evolution of the probability distribution when choosing the modality "at the center" compared to the choice of the modality "behind the steering wheel". It is not the distribution that user have to see in this graph but the distribution evolution).

- The impact of the technical target attribute on the perceptual attributes: According to the model we presented in Figure 4, the speedometer dial position has an impact on the dashboard "novelty perception" as well as on the "control comprehensibility". This model not only helps the design to identify the relevant relations between the technical target attribute and the other design attributes, but also allows him knowing how it impacts them. For instance, the model states that a dashboard whose speedometer dial is positioned at the center is perceived by customers as more novel than a dashboard whose speedometer dial is positioned behind the steering wheel. However, that choice deteriorates the control comprehensibility. In a sense, the model allows designer comparing the two possible technical choices related to the speedometer dial position (i.e. at the center or behind the steering wheel) in a multicriteria way (cf. Figure 6). 
W. Ben Ahmed, B. Yannou

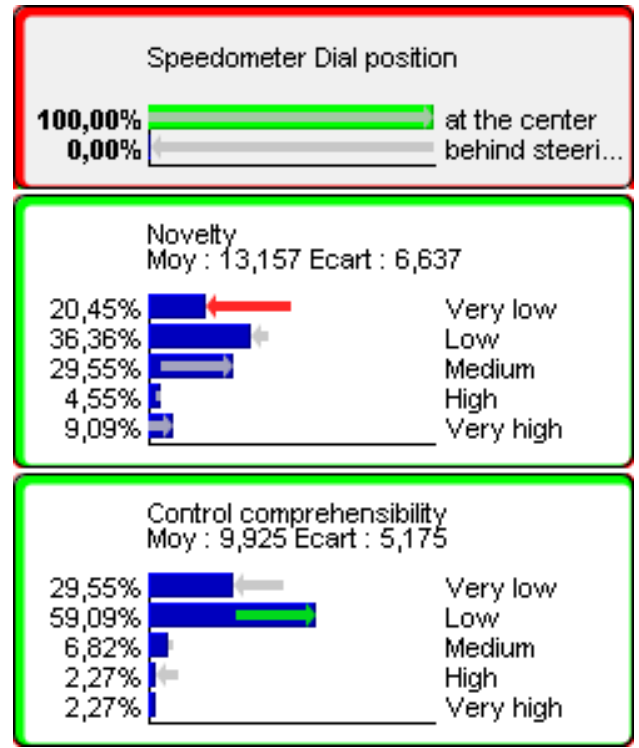

Figure 6 The influence of a technical choice "speedometer dial position= at the center" on the dashboard novelty lay out as well as on the control comprehensibility

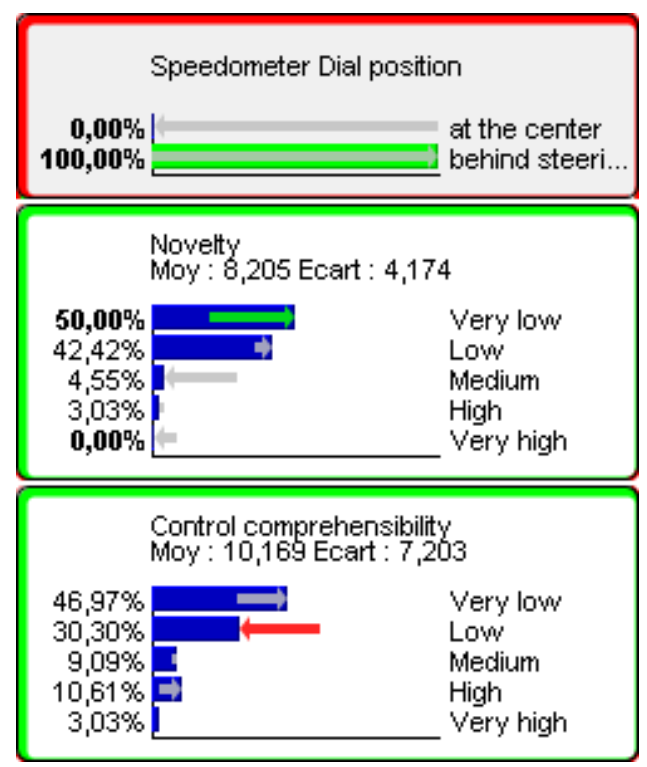

Figure 7 The influence of a technical choice "speedometer dial position= behind steering wheel" on the dashboard novelty lay out as well as on the control comprehensibility

The use of a supervised learnt model allows carrying out a local optimization: it concerns only the most correlated attributes to the target attribute. For example, choosing to position the speedometer dial at the center of the dashboard improves the novelty perception, but what about the impact of such a choice on all other attributes? A 


\section{A bayesian learning between perceptions and technical characteristics}

supervised learnt model does not allow answering such a question and that is why we propose in the next sub-section the use of an unsupervised learnt model.

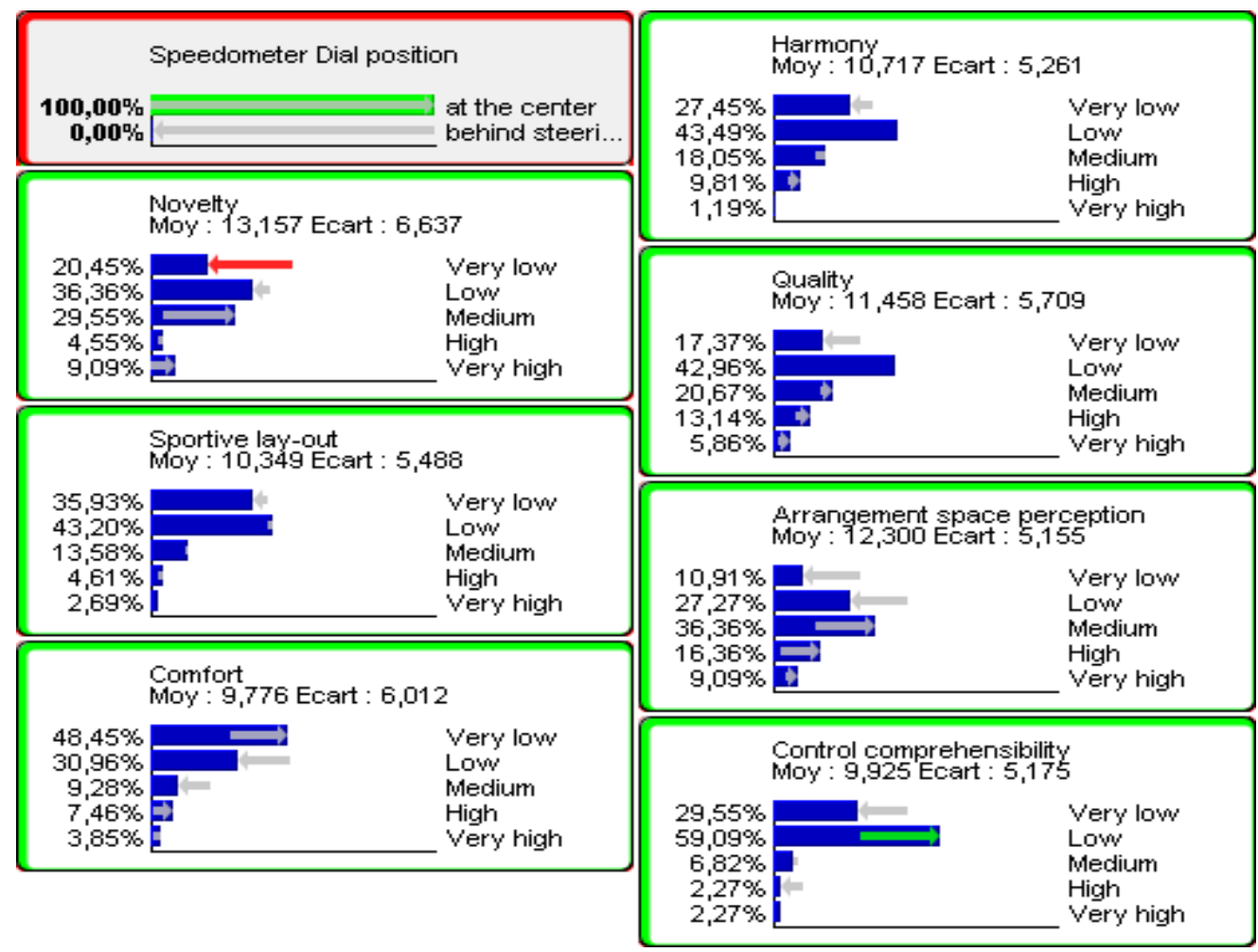

Figure 8 The influence of the speedometer dial position on the perceptual attributes characterizing a dashboard. According to this figure, placing the speedometer dial at the dashboard center improves the novelty perception, the sportive lay-out, the harmony perception, the quality perception, the arrangement space perception. But, at the same time this choice deteriorates the comfort perception

\section{(b) The use of an unsupervised learnt model in a direct scenario}

When a designer performs a choice concerning a given technical attribute, he may be interested by the information related to his choice impact on all attributes (technical as well as perceptual). Indeed, improving a given perceptual attribute may deteriorate another perceptual attribute and/or introduce hard technical constraints. This information is fundamental for a global optimization of the design. As we noticed in the previous section, a supervised learnt model does not allow obtaining such an information. To handle that issue, we propose the use of a non supervised model such the one we presented in Figure 2. Obviously, such a model is less precise than a supervised model, but it is more reliable for the global optimization to avoid erroneous decisions.

The interactive simulation of the model we presented in Figure 2 allows us having an idea about the global impact of the choice related to the speedometer dial position. According to Figure 8, locating the speedometer dial at the dashboard centre improves the novelty perception, the sportive lay-out, the harmony perception, the quality 
perception, the arrangement space perception. But, at the same time this choice deteriorates the comfort perception.

A technical choice may also be incompatible with other technical attributes or induce hard constraints toward other technical choices (e.g. cost, hard technology, functional incompatibility, etc.) (cf. Figure 9).

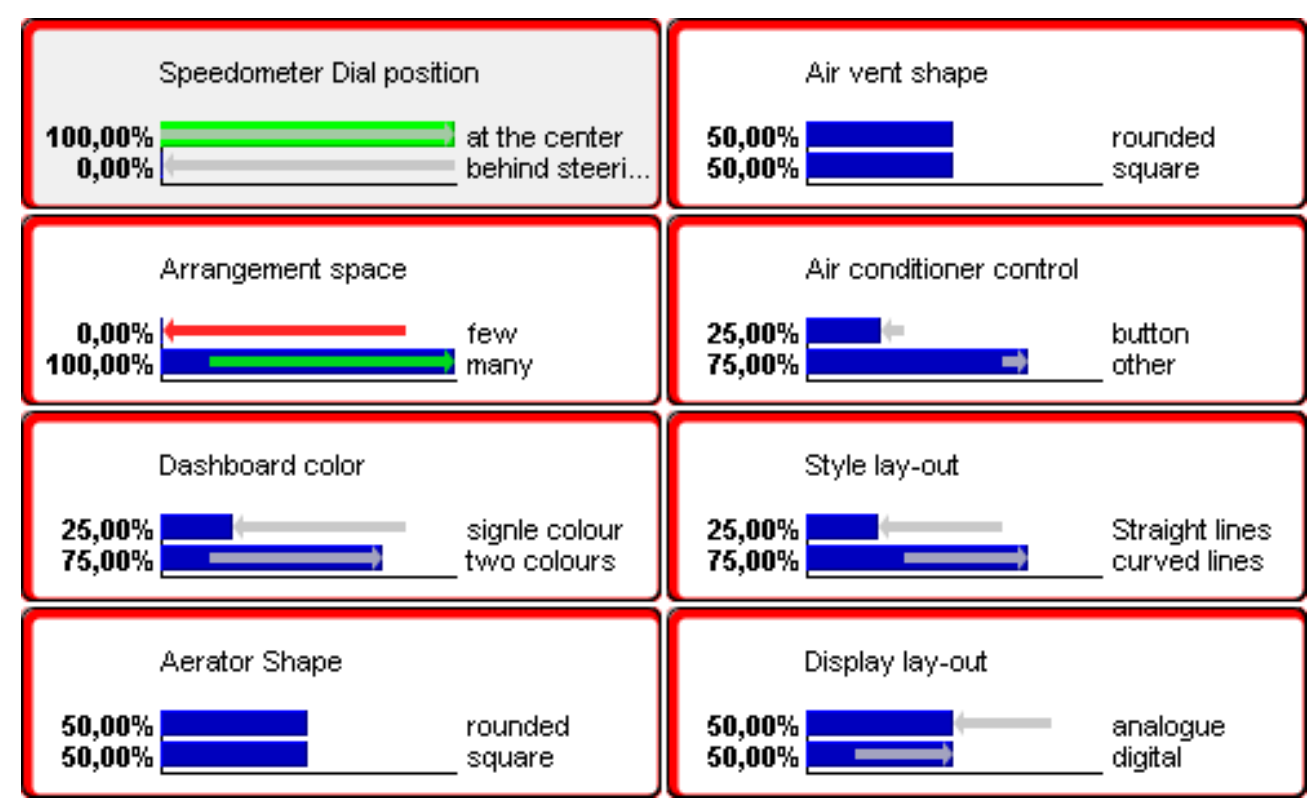

Figure 9 The interaction of the speedometer dial position with the technical attributes characterizing a dashboard. According to this figure, dashboards whose speedometer dial is located at the centre have generally many arrangement spaces, two colors, curved lines style lay-out and digital display lay-out ${ }^{1}$.

\subsubsection{Inverse scenario}

The inverse scenario allows answering the question "what are the best choices (related to technical attributes) the designer has to perform in order to maximize a perceptual attribute?". This is a typical question a designer asks when he carries out a design synthesis task. In the previous section we showed how a bayesian network allows a designer to simulate the impact of a technical choice on perceptual and technical attributes: input=design choice, output=impact on design attributes and performances. In this section, we show how the same model allows a designer identifying all possible

1 What user should interpret in Figure 9 is not the probability distribution itself, but its evolution compared to the opposite choice. For example, although

$P\left(\right.$ Display_lay-out $=$ analogue $/$ speedometer_position $\left.=a t \_t h e \_c e n t e r\right)=P($ Display_lay -

out $=$ digital/speedometer_position $=$ at the center $)$, what user should see is the fact that $P($ Display_lay-out $=$ analogue $/$ speedometer_position $=$ at_the_center $)>P($ Display_lay-

out=digital/speedometer_position = behind the steering wheel $)$. By the same interpretation rule, we can notice that the "Aerator shape" is independent from the "speedometer position". The arrows help user performing such interpretation task. 
A bayesian learning between perceptions and technical characteristics

design choices that allow him optimizing a given perceptual attribute (or performance): input=perceptual attribute to be optimized, output=possible design choices. In other words, this scenario consists in observing a perceptual attribute and analyzing how it interacts with the other attributes.

As an example, we take the "dashboard novelty perception" as target attribute to optimize and show how a BN allows identifying the best technical choices designers can perform to improve that attribute. Supervised and unsupervised learnt model can be used.

\section{(a) The use of a supervised learnt model in a inverse/synthesis scenario}

The supervised learned model we presented in Figure 3 states that the main relevant technical attributes that impact the novelty perception of a dashboard are the "speedometer dial position", the "dashboard color" and the "style lay out". Moreover, that model can suggest to the designer how to carry out the required adjustments (cf. Figure 10) to improve the dashboard novelty perception.

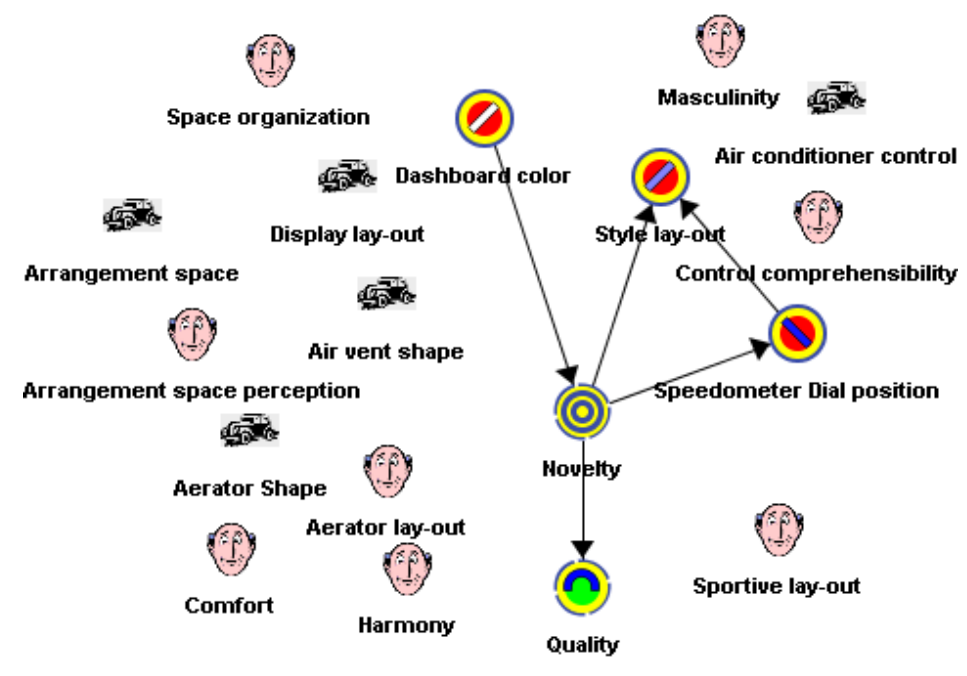

Figure 10 Supervised learning to characterize a target attribute (e.g. "Novelty") and to represent its sensitivity towards attributes impacting it.

According to Figure 10, to improve the novelty perception, a designer should choose the second modality of the style lay-out attribute (i.e. "curved lines" instead of "straight lines") and/or the second modality of the dashboard color (i.e. "two colors" instead of "single color") and/or the first modality of the speedometer dial position (i.e. "at the centre" instead of "behind steering wheel"). Figure 11 illustrates these statements. 


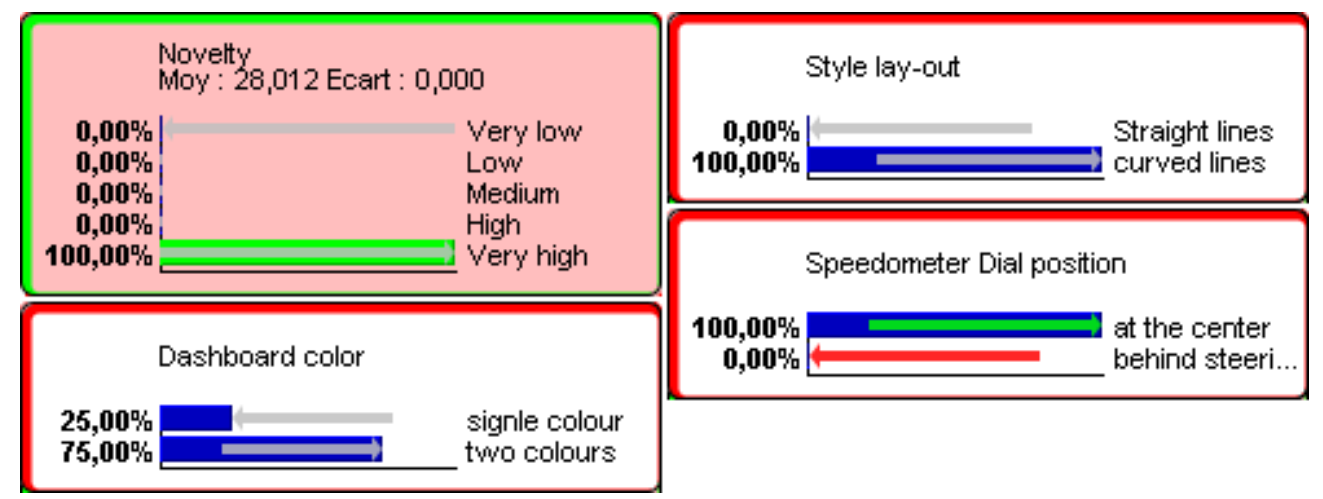

Figure 11 The optimal technical choices designer should carry out in order to improve the novelty perception. This figure confirms what Figure 10 states: i.e. to improve the novelty perception, designer should choose a dashboard having a curved lines style lay-out in stead of straight lines style lay-out and/or a two colors dashboard instead of a single color dashboard and/or a dashboard whose speedometer dial is at the centre instead of a dashboard whose speedometer is behind the steering wheel.

The use of the supervised model is not sufficient because of the two following reasons:

- The technical solutions suggested by a supervised model are certainly the most relevant to be tuned in order to optimize the target attribute. But, controlling them may be hard to carry out because of constraints related to cost, time and/or technological difficulties. Then, a designer has to find other levers even though these levers are less efficient (in term of their impact) than the ones identified by the supervised model.

- Improving a perceptual target attribute may deteriorate other perceptual attributes.

To handle those issues, we propose to use a supervised learnt model, which allows a designer carrying out a global simulation to analyze the impact of his choices (cf. next sub-section).

\section{(b) The use of an unsupervised learnt model in a inverse/synthesis scenario}

The same unsupervised model we presented in Figure 2 may be used to optimize a perceptual attribute. Such a model allows the two following points:

- Identifying all technical choices a designer can perform to improve the perceptual target attribute. Thereby, the designer can choose to tune the technical attributes, which are at the same time the most relevant (in term of their impact on the target attribute) and which engender the least constraints (in term of cost, time, technologies, etc.) (cf. Figure 12).

- Analyzing the impact of the optimization and avoid to deteriorate other perceptual performances of the design. For example, Figure 13 shows that improving the novelty perception is consistent with the improvement of other perceptual attributes such as the "quality", the "sportive lay-out", the "harmony", the "comfort". However, it may at the same time worsen the "control comprehensibility", the "space 
A bayesian learning between perceptions and technical characteristics

organization" and the "masculinity lay out" of a dashboard. Providing a designer with all these information is crucial to help him optimizing his choices.

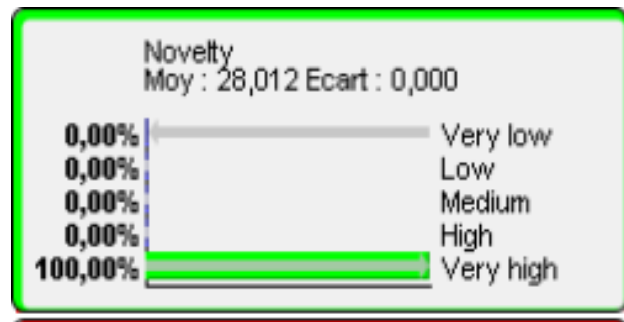

Speedometer Dial position

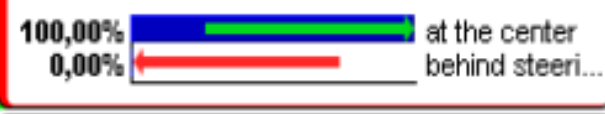

Dashboard color

$24,56 \%$

$75,44 \%$ two colours

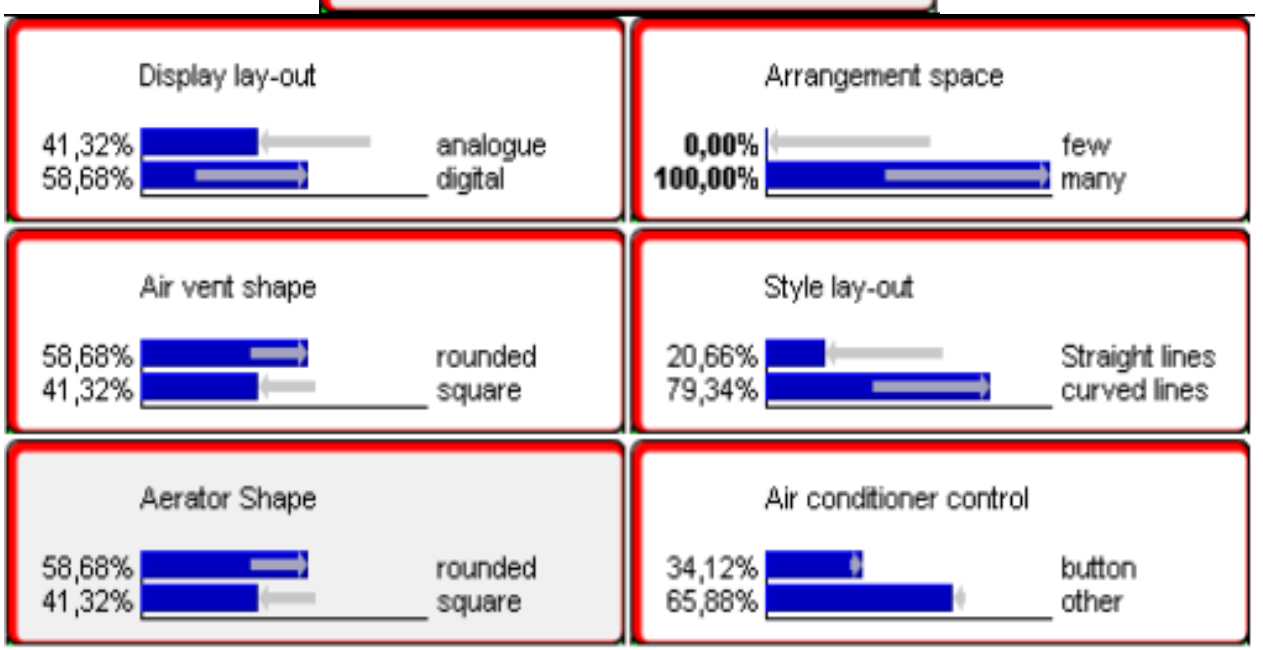

Figure 12 The optimal technical choices designer should carry out in order to improve the novelty perception of a dashboard. Compared to Figure 11, this model allows identifying all technical choices and not only the most relevant. Hence, it provides designer with more flexibility and choice possibilities. 
W. Ben Ahmed, B. Yannou

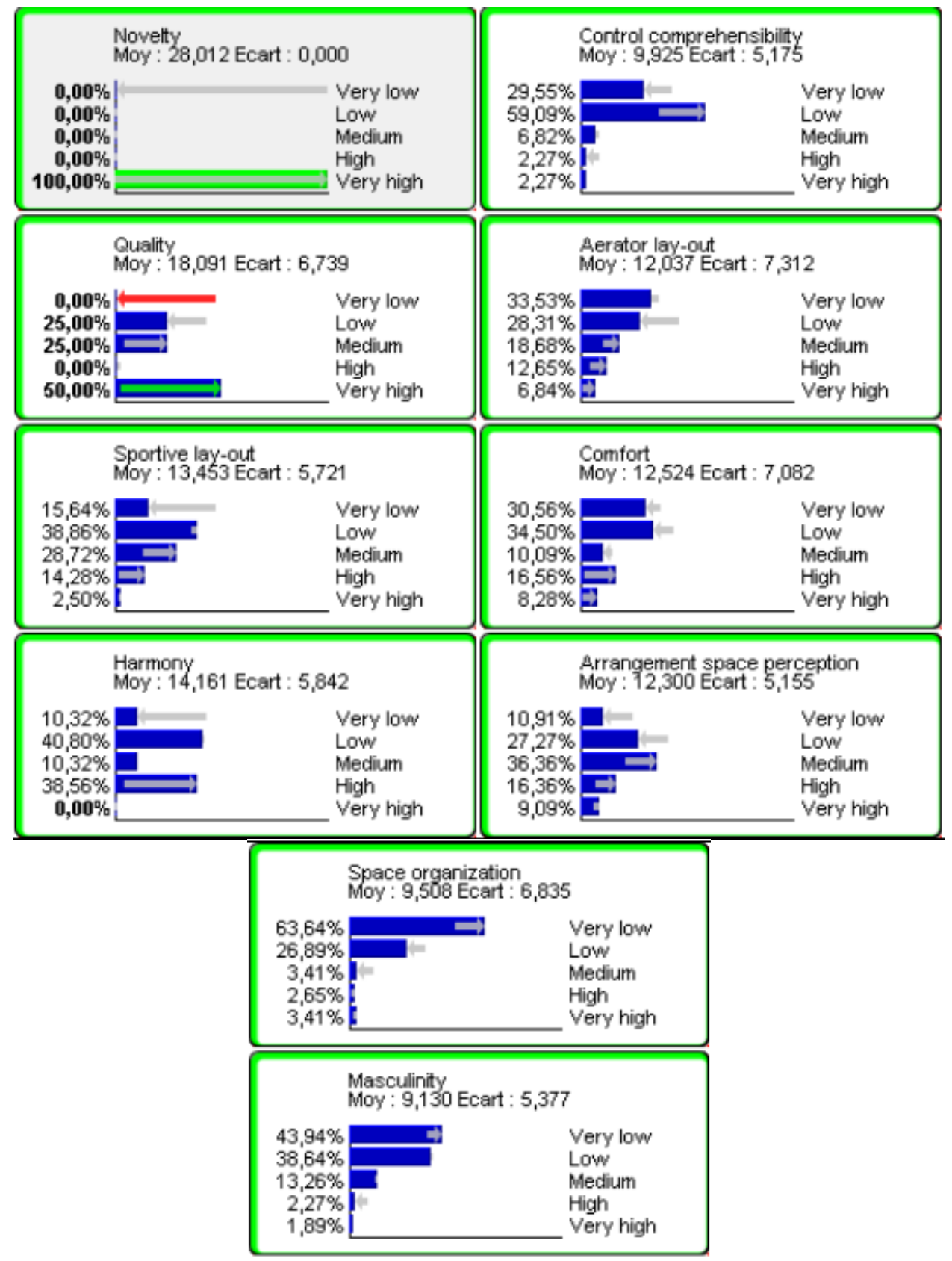

Figure 13 The impact of improving the novelty perception of a dashboard. Improving the novelty perception of a dashboard may be consistent with the improvement of other perceptual attributes (e.g. quality, harmony), but it may also deteriorate some other perceptual attributes (e.g. control comprehensibility, space organization). 
A bayesian learning between perceptions and technical characteristics

\subsubsection{A compound scenario for designing under constraints}

In a design process, a designer is confronted to many constraints, which make the control of some technical attributes hard (or impossible) because of restrictions related to the cost and/or time and/or technology. In that case, the designer has to freeze some technical choices and tune others. Suppose a designer looks for the different technical possibilities to improve the novelty perception of a dashboard he is developing and suppose he has no other choice but putting the speedometer dial behind the steering wheel. According to the previous sections (cf.

Figure 12), this constraint does not fit with his objective because a dashboard whose speedometer dial position is at the center looks more novel than a dashboard whose speedometer is behind the steering wheel. Thereby the designer must identify the other levers (i.e. the other technical choices) he can play with in order to improve the novelty perception of the dashboard he is designing as much as possible. The unsupervised model we presented in Figure 2 allows handling that issue.

In a sense, this is a mixed scenario of the two previous ones: at a first step a decision about a technical choice, so a direct scenario, is performed ("speedometer dial position" = "behind the steering wheel"). Then an inverse scenario is carried out to know what are the other technical attributes the designer can tune to optimize the perceptual attribute (i.e. to maximize the novelty perception). A simulation of a compound scenario is represented in Figure 14.

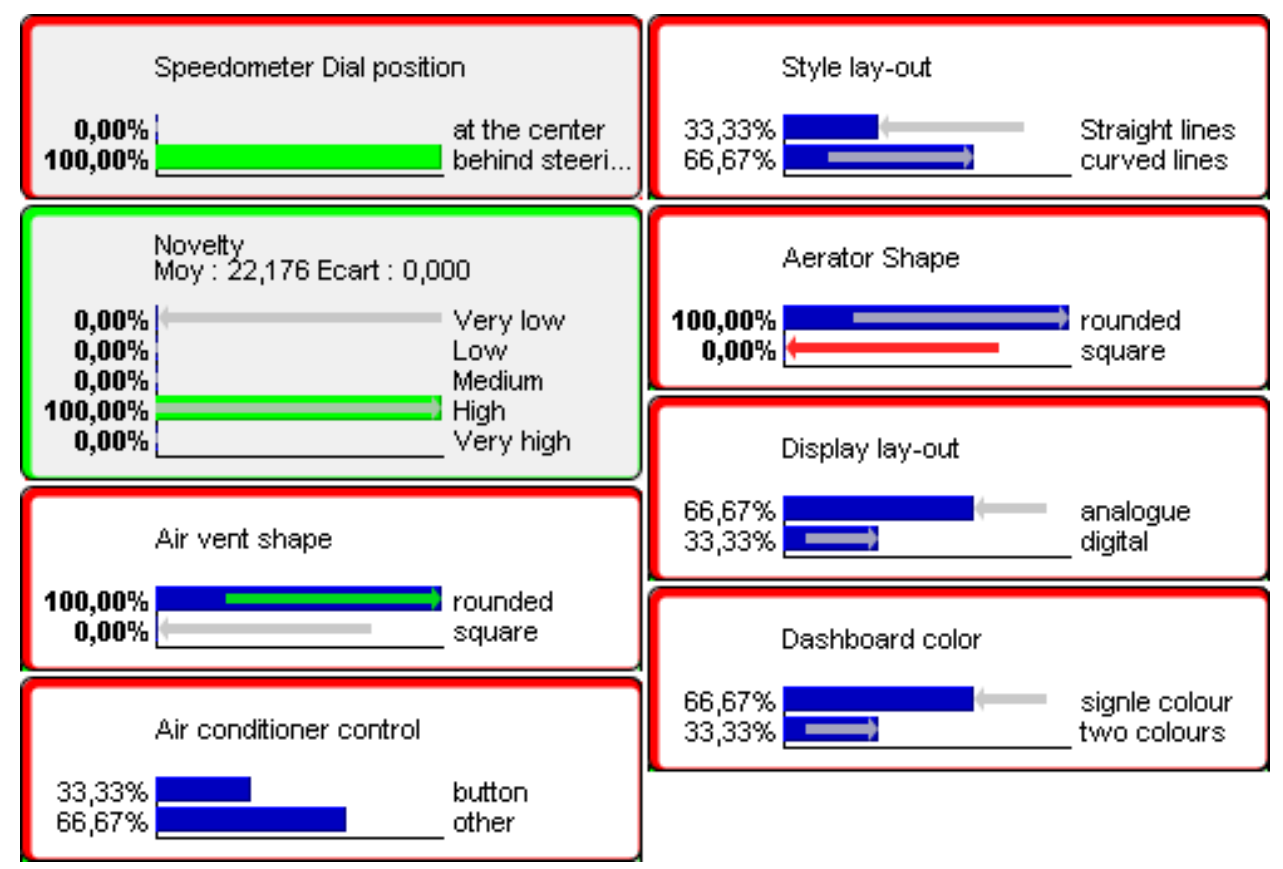

Figure 14 A compound scenario. A direct scenario is performed ("speedometer dial position" = "behind the steering wheel") and then an inverse scenario is carried out ("novelty perception" = "High") to know what are the other technical attributes the designer can tune to optimize the perceptual attribute (i.e. to maximize the novelty perception). 


\section{Discussion}

The main point discussed here is related to the reliability of our bayesian models. Since our approach to construct these BN models is a learning approach based on collected data, then the precision and reliability of these models essentially depend on the reliability and precision of the data used. Indeed, if the data used is not representative of reality, the reliability of the model may be deceptive. But, two considerations allow handling this drawback:

- The numerical assessment tools we propose here allow evaluating the accuracy of the model constructed and thereby to decide if some tasks have to be reconsidered such as the data pre-processing, the choice of a score function, the choice of the structure search strategy.

- The expert can implement his knowledge to refine the model automatically constructed especially in the data-preprocessing step when he discretizes the attributes.

- Another lever to handle the issue of data incompleteness is the possibility to have a larger data set, more representative than the one we used. Practically, if a larger number of dashboards are evaluated and a larger number of customers participate in the evaluation of these dashboards, the data collected would be more representative and thereby the model constructed would be more reliable.

- These models are evolutive: customer perception of a given design remains subjective (despite the non hedonistic condition, the assessments between customers may largely differ) and thereby evolves in time and depends on several parameters such as culture, geography, etc. Hence, data have to be updated so as to take that evolutivity into account. Since our models are automatically based on these data, their update (i.e. the update of the relations between attributes and of conditional probability tables) is then automatic.

Finally, the BN models we presented in this paper were presented to experts and their simulation results were judged very satisfactory.

\section{Conclusion and perspectives}

In a design process, designers need tools to help them understanding customers' needs and thereby predicting their appreciation level of a new product. In this paper, we propose two complementary types of bayesian network models to answer that question:

- Unsupervised learnt models: these models represent the whole set of probabilistic relationships existing within the data. They allow having an idea about all relationships that hold in the data set and thereby they are more efficient to carry out a global optimization of the design.

- Supervised learnt models: instead of learning a BN representing all the probabilistic relations that hold in the data set, unsupervised models allow refining the characterization of a specific target attribute in that they allow identifying the attributes that really characterize the target attribute. A target attribute may be a 
A bayesian learning between perceptions and technical characteristics

technical attribute as well as a perceptual attribute. These models are more efficient to carry out a local optimization of the design.

In this paper, we defined three scenarios to use our models:

- A direct/analysis scenario in which the input is a technical attribute and the output consists of an analysis of its impacts on the other design attributes (technical and perceptual). This scenario is typically used when a designer wants to compare different technical solutions.

- An inverse/synthesis scenario in which the input is a perceptual attribute and the output consists of a list of technical choices the designer can perform to optimize the perceptual attribute. This scenario is typically used when a designer wants to identify all technical solutions to optimize a given performance of his design. He can then choose the most relevant and the least constraining technical attributes to tune.

- A compound scenario in which both technical and perceptual attributes are observed and the impact on the other attributes is analyzed. This scenario is typically used when a designer wants to optimize a given performance of his design under design constraints (cost, time, technology, etc.).

Finally Bayesian Networks turn out to be a very flexible and powerful technology in preliminary perceptual (or emotional) design in terms of simulation and prediction capacities.

\section{Acknowledgements}

We would like to gratefully thank Anthony Harvey and Olaf Mahlo who have organized and carefully completed the evaluation workshop on the dashboards during their MSc research internship.

\section{References}

Akaike, H. (1974) 'A new look at the statistical model identification', IEEE Transactions on Automatic Control, Vol. 19, No. 6, pp.716-723.

BayesiaLab (2006) In Secondary 'Bayesia tutorial Book', available on www.bayesialab.com.

Bouckaert, R. R. (1993) 'Probabilistic network construction using the minimum descriptions length principle', Proceedings of the European Conference on Symbolic and Quantitative Approaches to Reasoning and Uncertainty.

Buntine, W. (1996) 'A guide to the literature on learning graphical models', IEEE Transactions on Knowledge and Data Engineering, Vol. 8, pp.195-210.

Chickering, D. M. (2002a) 'Learning equivalent classes of bayesian network structures', Journal of Machine Learning Research, Vol. 2, pp.445-498.

Chickering, D. M. (2002b) 'Optimal structure identification with greedy search', Journal of Machine Learning Research, Vol. 3, pp.507-554.

Cooper, G. F. and Herskovits, E. (1992) 'A bayesian method for the induction of probabilistic networks from data', Machine Learning, Vol. 9, No. 309. 
W. Ben Ahmed, B. Yannou

Friedman, N. (1998) 'The bayesian structural EM algorithm', Proceedings of the Fourteenth Uncertainty in Artificial Intelligence Conference (UAI-1998), San Francisco, CA, Morgan Kaufmann Publishers, pp.129-138.

Friedman, N. and Koller, D. (2003) 'Being bayesian about network structure. A bayesian approach to structure discovery in bayesian networks', Machine Learning, Vol. 50, pp.95-125.

Harvey, A. (2005) In Secondary 'Application of an integrated method to a study of the consumer perceptions of automobile dashboards'Research Internship report in Ecole Centrale Paris, University of Bath.

Heckerman, D., Geiger, D. and Chickering, D. M. (1995) 'Learning Bayesian networks: the combination of knowledge and statistical data', Machine Learning, Vol. 20, No. 3, pp.197-243.

Hsiao, S. W. and Wang, H. P. (1998) 'Applying the semantic transformation method to product form design', Design Studies, Vol. 19, pp.309-330.

Huang, C. and Dawiche, A. (1996) 'Inference in Belief Networks: A Procedural Guide', International Journal of Approximate Reasoning, Vol. 15, pp.225-263.

Jensen, F. V. (1996) An Introduction to Bayesian Networks, UCL Press, London.

Jindo, T. and Hirasago, K. (1997) 'Application studies to car interior of Kansei engineering', International Journal of Industrial Ergonomics, Vol. 19, pp.105114.

Jordan, M. I. (1998) Learning in Graphical Models, MIT Press, Cambridge, Massachusetts.

Jouffe, L. (2002) 'Nouvelle classe de méthodes d'apprentissage de réseaux bayésiens', Proceedings of the Journées francophones d'Extraction et de Gestion des Connaissances (EGC), Montpellier, France.

Jouffe, L. and Munteanu, P. (2001) 'New Search Strategies for Learning Bayesian Networks', Proceedings of the Tenth International Symposium on Applied Stochastic Models, Data Analysis, Compiègne, France.

Lam, W. and Bacchus, F. (1994) 'Learning bayesian belief networks: An approach based on the MDL principle', Computational Intelligence, Vol. 10, pp.269-293.

Limayem, F. and Yannou, B. (2004) 'Generalization of the RCGM and LSLR Pairwise Comparison Methods', Computers and Mathematics with Applications, Vol. 48, pp.539-548.

Meek, C. (1997), 'Graphical Models: Selecting causal and statistical models', PhD thesis, Carnegie Mellon University.

Mehmet, K. and Gregory, C. (2002) 'A bayesian network scoring metric that is based on globally uniform parameter priors', Proceedings of the 18th Annual Conference on Uncertainty in Artificial Intelligence (UAI-02), San Francisco, CA, Morgan Kaufmann Publishers, pp. 251-258.

Munteanu, P. and Bendou, M. (2001) 'The EQ Framework for Learning Equivalence Classes of Bayesian Networks', Proceedings of the First IEEE International Conference on Data Mining (IEEE ICDM), San José.

Nagamachi, M. (1995) 'Kansei Engineering: A new ergonomic consumer-oriented technology for product development', International Journal of Industrial Ergonomics, Vol. 15, pp.3-11.

Nagamachi, M. (2002) 'Kansei engineering as a powerful consumer-oriented technology for product development', Applied Ergonomics, Vol. 33, pp.289-294. 
A bayesian learning between perceptions and technical characteristics

Naïm, P., Wuillemin, P. H., Leray, P., Pourret, O. and Becker, A. (1999) Les Réseaux Bayésiens, Eyrolles, Paris.

Neapolitan, R. E. (2003) Learning Bayesian networks, Prentice Hall, Upper Saddle River, NJ.

Petiot, J.-F. and Yannou, B. (2004) 'Measuring consumer perceptions for a better comprehension, specification and assessment of product semantics', International Journal of Industrial Ergonomics, Vol. 33, No. 6, pp.507-525.

Rissanen, J. (1986) 'Stochastic complexity and modeling', The Annals of Statistics, Vol. 14, No. 3, pp.1080-1100.

Robinson, R. (1977) 'Counting unlabeled acyclic diagraphs' In Combinatory Mathematics $V($ Ed, Little, C.) Springer, Berlin, pp.28-43.

Schwarz, G. (1978) 'Estimating the dimension of a model', Annals of Statistics, Vol. 6, pp.461-464.

Spirtes, P., Glymour, C. and Scheines, R. (2000) Causation, Prediction, and Search, MIT Press, 2nd edition, Cambridge, MA.

Suzuki, J. (1993) 'A construction of bayesian networks from databases based on an MDL principle', Proceedings of the 9th Annual Conference on Uncertainty in Artificial Intelligence (UAI-93), San Francisco, CA, Morgan Kaufmann Publishers.

Yannou, B. (2007) 'Easy and flexible specifications and product evaluations by expert and customer comparisons with existing products', Submitted to the International Conference on Engineering Design: ICED'07, Cité des Sciences et de l'Industrie, Paris, France, August 28-31.

Yannou, B. and Petiot, J.-F. (2004) 'A methodology for integrating the customers' assessments during the conceptual design', Proceedings of the DETC/DTM: ASME Design Engineering Technical Conferences / Design Theories and Methodologies, Salt Lake City, Utah, USA, Sept. 28 - Oct. 2. 